\title{
Characterization of calorimeter responses under laboratory conditions thanks to an optimized transient thermal test bench
}

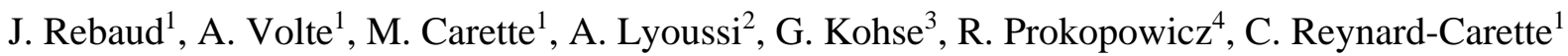 \\ ${ }^{1}$ Aix Marseille Univ, Université de Toulon, CNRS, IM2NP, Marseille, France \\ ${ }^{2}$ CEA/DES/IRESNE/DER, Section of Experimental Physics, Safety Tests and Instrumentation, \\ Cadarache, F-13108, Saint Paul-lez-Durance, France \\ ${ }^{3}$ Massachusetts Institute of Technology, Nuclear Reactor Laboratory, Cambridge, Massachusetts, USA \\ ${ }^{4}$ National Centre for Nuclear Research, Otwock, Poland
}

Corresponding author: jeremy.rebaud@etu.univ-amu.fr

\begin{abstract}
This paper presents an experimental study of the calibration of a non-adiabatic single-cell calorimeter called KAROLINA. This type of sensor is used to quantify the nuclear heating rate inside MTRs (Material Testing Reactors). The calibration of this sensor type is carried out in laboratory conditions without irradiation. The calibration corresponds to a preliminary step, which is crucial to use this sensor in real conditions. In the case of the studied single-cell calorimeter owning no heating element, the calibration is realized with a specific test bench, called BERTAN, based on thermal transient regimes. First of all, the paper describes the methodology of the determination of the calorimeter sensitivity and the dedicated experimental set-up developed recently. Then the paper is devoted to a parametric study carried out on the KAROLINA single-cell calorimeter to determine the influence of several parameters on its thermal time constant (temperature, velocity, phase, method).
\end{abstract}

Keywords - Nuclear Heating Rate, Calorimetry, Calibration

\section{INTRODUCTION}

$\mathrm{T}$ he design of future nuclear power reactors requires the study and understanding of the behavior of advanced material and fuel expected to be used within. One of the means of study is the use of Material Testing Reactors. These reactors, specially dedicated to research, allow the study of the ageing of the materials, the nuclear fuel behavior but also the testing of innovative in-pile sensors. These sensors are required to quantify accurately online key quantities in order to lead to the best understanding of nuclear phenomena. The nuclear heating rate is one of these key quantities. It corresponds to an energy deposition rate per unit of mass $\left(\mathrm{W} . \mathrm{g}^{-1}\right)$ and is measured with heat-flow calorimeters. This type of non-adiabatic sensors is based on the measurement of temperature increase due to the large amount of energy deposition induced by the radiationmatter interactions. There are two families of these sensors [1]: the differential calorimeter [2-4] and the single-cell calorimeter
[5]. There are many similarities between these two families: a sample, an inert gas inside a jacket, and K-type thermocouple(s). However, there are two main differences: the first one is the number of cells that composes the sensor (one for the single-cell calorimeters and several for the differential calorimeters (sample cell(s) and reference(s))), and the second one, is the presence of heating elements inside the differential calorimeters. These differences lead to advantages and drawbacks of use for each family (cf. Table I).

TABLE I: ADVANTAGES IN GREEN AND DRAWBACKS IN RED OF TWO HEATFLOW CALORIMETER FAMILIES

\begin{tabular}{|c|c|c|}
\hline & Differential calorimeter & Single-cell calorimeter \\
\hline Size & $\geq 200 \mathrm{~mm}$ & $\leq 72 \mathrm{~mm}$ \\
\hline Wire number & $\geq 9$ & $\leq 2$ \\
\hline $\begin{array}{l}\text { Measurement } \\
\text { methods }\end{array}$ & 3 & 1 \\
\hline Range & $\leq 20 \mathrm{~W} \cdot \mathrm{g}^{-1}$ & $\leq 20 \mathrm{~W} \mathrm{~g}^{-1}$ \\
\hline $\begin{array}{l}\text { Measurement } \\
\text { Time }\end{array}$ & $\begin{array}{c}\text { Response time } \\
+ \text { Stabilization time } \\
+ \text { One displacement at least }\end{array}$ & $\begin{array}{c}\text { Response time } \\
+ \text { Stabilization time }\end{array}$ \\
\hline $\begin{array}{l}\text { Sample } \\
\text { height }\end{array}$ & $\leq 20 \mathrm{~mm}$ & $\geq 15 \mathrm{~mm}$ \\
\hline $\begin{array}{l}\text { Calibration } \\
\text { methods }\end{array}$ & Steady & Transient \\
\hline
\end{tabular}

Because of these different designs, there are two different calibration methods (cf. Fig. 1) [6]. Indeed, to calibrate the differential calorimeter, an electrical current is injected into each heating element in order to generate a Joule effect, which will allow the simulation of the nuclear heating rate. The lack of heating elements inside the single-cell calorimeters requires another calibration method. This other calibration method is based on a thermal transient protocol for which the calorimeter passes rapidly from a medium 1 at a temperature 1 to a medium 2 at a temperature 2 (cf. Fig. 1). 

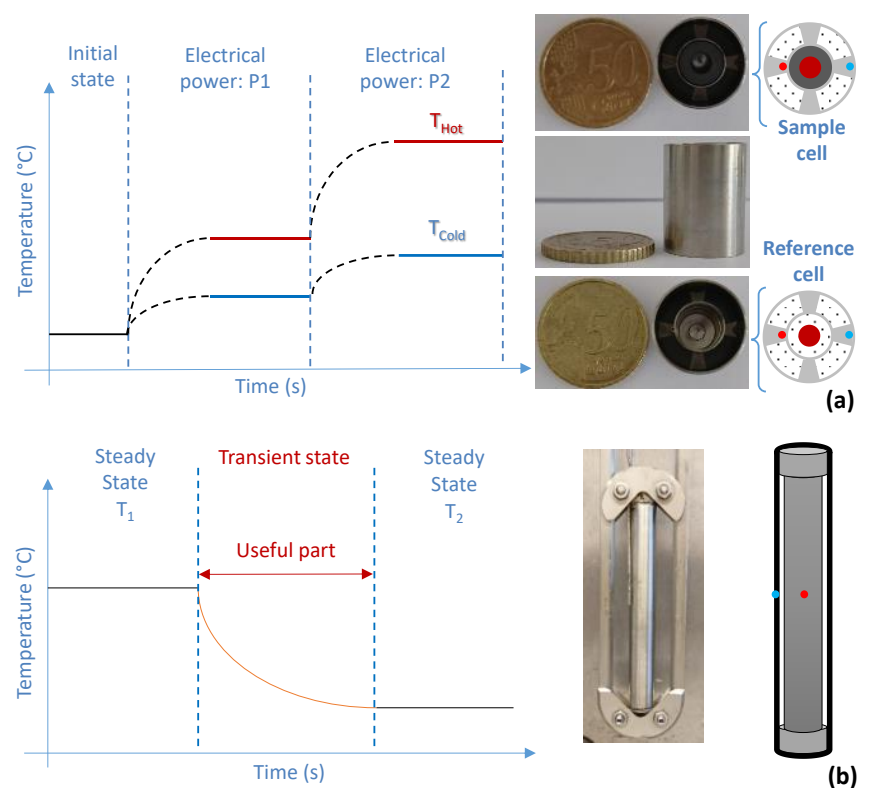

Fig. 1 Calibration protocols for: (a) Differential calorimeters; (b) Single-cell calorimeters.

The calibration protocol for the single-cell calorimeters under laboratory conditions was first implemented with a manual transfer of the calorimeter from an oven to a water bath. This method has three main drawbacks. Firstly, it does not allow reproducible tests. Secondly, it leads to a transfer time that can be long. Lastly due to the test protocol, only one type of transient state is exploitable (hot source to cold source) [7]. The automation and improvements of this calibration method led to the design of the BERTRAN test bench, which gave conclusive preliminary results [8]. Consequently, a new test campaign was then set up in order to study the calibration of a single-cell calorimeter called KAROLINA with various controlled experimental conditions.

The paper will focus on this parametrical study. More precisely this paper will first describe the single-cell calorimeter KAROLINA. Then it will present the methodology for the determination of the thermal time constant $(\mathrm{k})$ and thus the sensitivity of the calorimeter. Afterwards, it will detail the BERTRAN bench used for the experimental tests, and the applied protocol. Finally, the parametric study will be shown and the analysis of the results for a test campaign will be discussed in order to define the influent factors on the thermal time constant value.

\section{KAROLINA CALORIMETER}

The KAROLINA calorimeter is a single-cell calorimeter developed by the National Center for Nuclear Research (NCBJ) to carry out experiments for the mapping of nuclear heating rate under real conditions in the MARIA research reactor [9].

The calorimeter shape is a cylinder with a height of $72 \mathrm{~mm}$ and an external diameter of $9.4 \mathrm{~mm}$ (cf. Fig. 2). A graphite sample is located in the center of this calorimeter, surrounded by a thin layer of inert gas in order to thermally isolate the sample from the outside environment and to impose a specific sensitivity. This sample is held in the center with two sample holders made of zirconium oxide which have a porous composition in order to minimize their impact by having a thermal conductivity close to the gas. The assembly is packaged in a stainless-steel jacket with an external diameter of $9.4 \mathrm{~mm}$ and a thickness of $0.5 \mathrm{~mm}$. Regarding the instrumentation of this sensor, it has two K-type thermocouples of $0.5 \mathrm{~mm}$ diameter. The two thermocouples are located at the same height. The first one is in the middle of the sample (named sample thermocouple), and the second one is welded on the outer wall of the jacket (named fluid thermocouple).

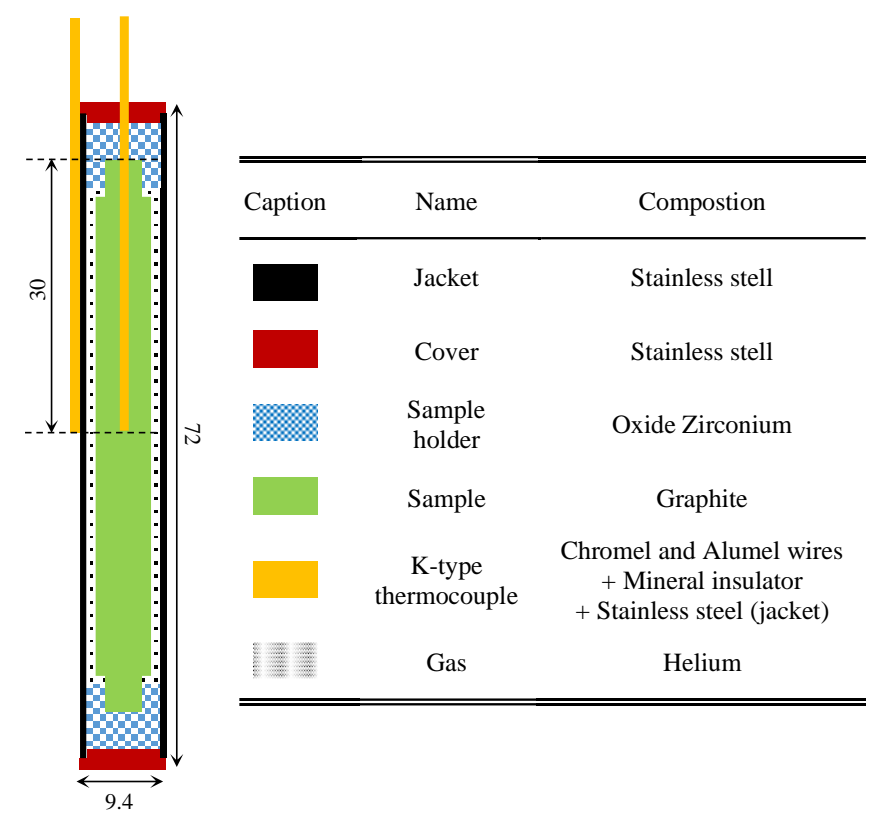

Fig. 2 Scheme of KAROLINA single-cell calorimeter (dimensions expressed in millimeters).

III. METHODOLOGY FOR THE DETERMINATION OF THE SENSITIVITY OF A SINGLE-CELL CALORIMETER

A 1-D theoretical study of the KAROLINA calorimeter response, built thanks to a thermal balance taking into account some hypotheses, allows the determination of the temperature of the calorimeter sample versus time under irradiation conditions.

The hypotheses are:

- No thermal radiation,

- Heat exchange only on the radial axis,

- Isothermal sample,

- Isothermal wall,

- No contact thermal resistance,

- Energy deposition only into the sample.

The heat balance applied on the sample is given by this equation (1) (the parameters and their units are in Table II):

$$
m_{s} E_{n}=\rho C_{p} V \frac{\partial T_{s}}{\partial t}+\left(\frac{T_{s}-T_{f}}{R}\right)
$$

The resolution of this equation leads to the temporal response of the calorimeter under irradiation conditions (2):

$$
T_{s}(t)=T_{f}+E_{n} \frac{k}{C_{p}}+\left(T_{s_{0}}-T_{f}-E_{n} \frac{k}{C_{p}}\right) e^{-\frac{t}{k}}
$$


with $k=R \rho C_{p} V$ which corresponds to the calorimeter thermal time constant and $T_{S_{0}}$ the initial sample temperature.

When the steady state is reached for a given nuclear heating rate $(\mathrm{t} \rightarrow+\infty)$ at a specific location inside the reactor, the expression (2) becomes:

$$
E_{n}=\frac{\left(T_{s_{+\infty}}-T_{f}\right) C_{p}}{k}
$$

Consequently, the nuclear heating rate can be deduced from a measurement of steady temperatures and knowing its sensitivity which is equal to:

$$
S=\frac{k}{C_{p}}
$$

TABLE II

UNITS AND SYMBOLS FOR USED PARAMETERS

\begin{tabular}{ccc}
\hline \hline Symbol & Quantity & Unit \\
\hline $\mathrm{m}_{\mathrm{s}}$ & Sample mass & $\mathrm{g}$ \\
$\mathrm{E}_{\mathrm{n}}$ & Nuclear Heating rate & $\mathrm{W} \cdot \mathrm{g}^{-1}$ \\
$\rho$ & Sample density & $\mathrm{kg} \cdot \mathrm{m}^{-3}$ \\
$\mathrm{C}_{\mathrm{p}}$ & Sample specific heat & $\mathrm{J} \cdot \mathrm{kg}^{-1} \cdot \mathrm{K}^{-1}$ \\
$\mathrm{~V}$ & Sample volume & $\mathrm{m}^{3}$ \\
$\mathrm{~T}_{\mathrm{f}}$ & Fluid temperature & $\mathrm{K}$ \\
$\mathrm{T}_{\mathrm{s}}$ & Sample temperature & $\mathrm{K}$ \\
$\mathrm{R}$ & Total thermal resistance between & $\mathrm{K} . \mathrm{W}^{-1}$ \\
$\mathrm{t}$ & the sample and the fluid & $\mathrm{s}$ \\
$\mathrm{k}$ & Time & $\mathrm{s}$ \\
$\mathrm{S}$ & Thermal time constant & $\mathrm{kg} \cdot \mathrm{K} . \mathrm{W}^{-1}$ \\
\hline \hline
\end{tabular}

The value of the specific heat of the sample versus the temperature can be determined by using a Differential Scanning Calorimeter [10]. The thermal time constant can be measured under laboratory conditions thanks to a thermal transient protocol moving the calorimeter from a bath $\mathrm{N}^{\circ} 1$ with a temperature $\mathrm{T}_{1}$ to a bath $\mathrm{N}^{\circ} 2$ with another temperature $\mathrm{T}_{2}$. Under laboratory conditions, out-of-pile, there is no nuclear heating rate in the sample $\left(E_{n}=0\right)$ and consequently the equation 1 becomes:

$$
\rho C_{p} V \frac{\partial T_{s}}{\partial t}+\left(\frac{T_{s}-T_{f}}{R}\right)=0
$$

At the beginning of the experiments when the calorimeter is immersed in the bath $\mathrm{N}^{\circ} 2$ at $\mathrm{t}=\mathrm{t}_{1}$, the calorimeter sample temperature is equal to $\mathrm{T}_{\mathrm{s} 1}$. The resolution of the equation (3) by taking this initial condition into account leads to this expression (6):

$$
\frac{T_{s}-T_{f}}{T_{s_{1}}-T_{f}}=e^{-\frac{t}{k}+\frac{t_{1}}{k}}
$$

The expression (6) can be transformed:

$$
\ln \left(\frac{T_{s}-T_{f}}{T_{S_{1}}-T_{f}}\right)=-\frac{t}{k}+\frac{t_{1}}{k}
$$

Two values of $\mathrm{k}$ can be deduced by plotting this expression (7) with the temporal results in temperature (cf. Fig. 3) and by using a linear fit to calculate the slope $\left(a=-\frac{1}{k}\right)$ and the $\mathrm{y}$-intercept $\left(b=\frac{t_{1}}{k}\right)$.

Another method is to plot directly the sample temperature versus time. The thermal time constant corresponds to the response time of the sensor at $1 \tau(63 \%)$ or one third of the response time at $3 \tau(95 \%)$.

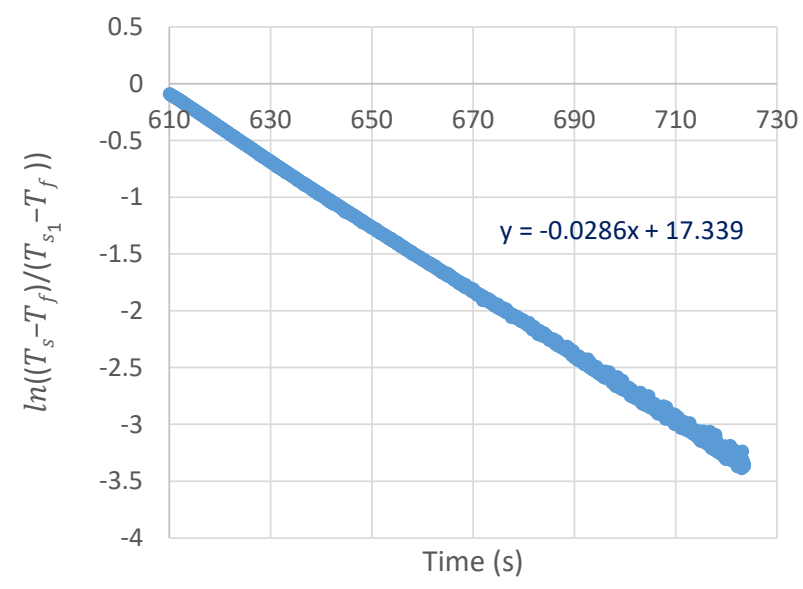

Fig. 3 Temporal curve representing the linearization of the transient state during the heating phase step with a propeller velocity of $1000 \mathrm{rpm}$ and with hot and cold temperatures of $250{ }^{\circ} \mathrm{C}$ and of $31^{\circ} \mathrm{C}$ respectively.

\section{PRESENTAtion OF ThE TEST BENCH AND THE OPERATING PROCEDURE.}

The calibration of the single-cell calorimeter KAROLINA has been performed with a test bench, named BERTRAN, specially designed to apply a thermal transient protocol in an automated way.

\section{A. BEnch Regime TRANsient (BERTRAN)}

The purpose of the BERTRAN test bench is to determine the transient response of the calorimeter when it changes rapidly from boundary conditions in temperature (from hot temperature to cold temperature or vice-versa). For this, it is necessary to have two media at two different temperatures and a mechanism to transfer the sensor automatically between these two media. These media are generated by means of two thermostatic baths filled with silicone oil. The silicone oil is chosen as heat transfer fluid because it as can be used up to $250{ }^{\circ} \mathrm{C}$ at atmospheric pressure. The two thermostatic baths are dedicated to having a cold environment (called cold bath) and a hot environment (hot bath) respectively.

The transfer mechanism is composed of a rail on which a pole is fixed and a mechanical drive system. At the end of the pole is put the KAROLINA calorimeter. An infrared sensor allows the control of the calorimeter position and its transfer. The transfer time of the calorimeter is around $1.5 \mathrm{~s}$.

These main elements of the BERTRAN bench are given in Figure 4. 


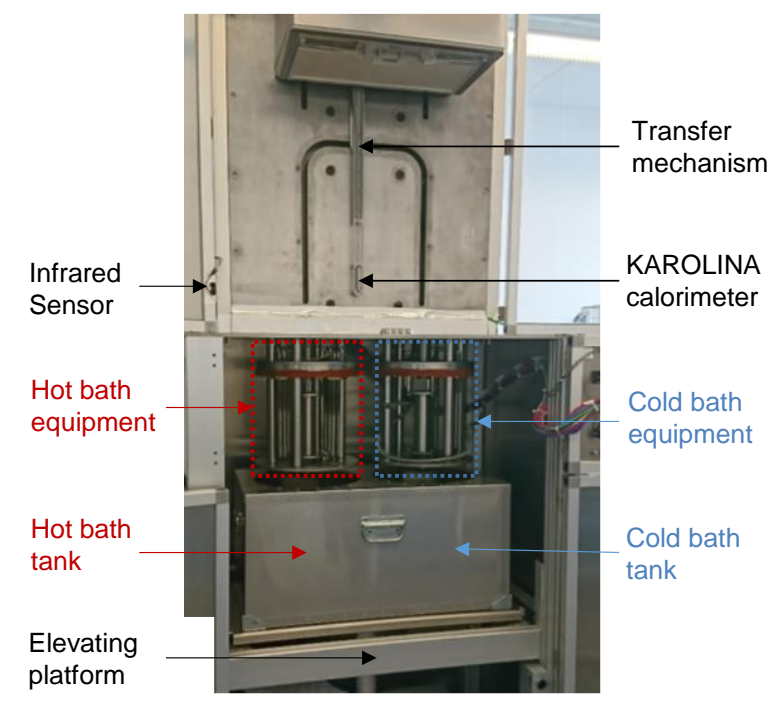

Fig. 4 Picture of calibration bench BERTRAN.

Each thermostatic bath has two parts (cf. Fig. 4 and Fig. 5). On the one hand, there is a silicon oil tank and on the other hand, there is an equipment, which allows the heating or the cooling of the silicone oil, the temperature stabilization and homogenization, and the generation of a downward forced convection.

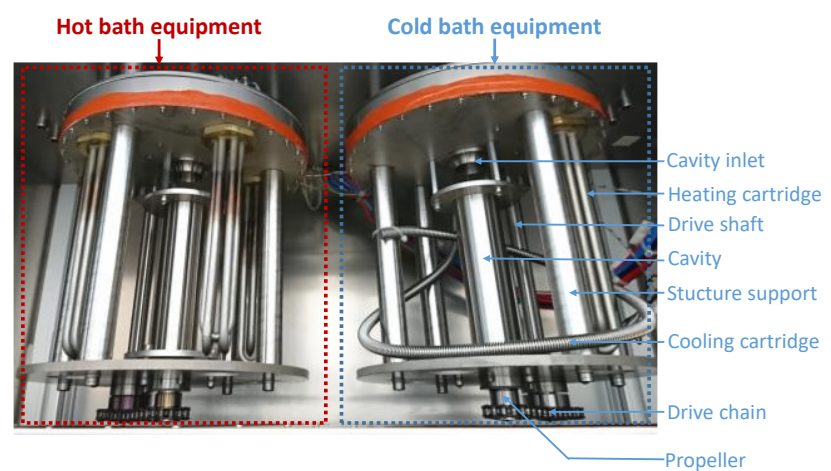

Fig. 5 Picture of the two bath-systems.

The two bath-systems are quite similar. They differ only in the elements within their temperature range ( 3 heating cartridges for the hot bath against a heating cartridge and a coil connected to a cryostat for the cold bath). These elements associated to temperature sensors and regulators allow the regulation of the bath temperature to reach a steady state in each bath. Moreover, in the center of each bath is placed a cylindrical cavity (vein) which simulates an experimental channel of reactor by its inner diameter of $34 \mathrm{~mm}$. At the bottom of each vein is located a propeller which causes a downward fluid flow in order to impose similar velocity and heat exchange conditions than those occurring in an experimental channel. In each vein there is a K-type thermocouple to control the temperature of the fluid flow in the vicinity of the sensor.
TABLE III: ACQUISITION CHANNELS OF BERTRAN BENCH

\begin{tabular}{cccc}
\hline \hline & Sensors & Quantity & Location \\
\hline $\begin{array}{c}\text { Channel } \\
1\end{array}$ & K-type & Temperature & KAROLINA external \\
thermocouple & $\left({ }^{\circ} \mathrm{C}\right)$ & jacket \\
Channel & K-type & Temperature & KAROLINA sample \\
2 & thermocouple & $\left({ }^{\circ} \mathrm{C}\right)$ & \\
Channel & K-type & Temperature & Cold bath vein \\
3 & thermocouple & $\left({ }^{\circ} \mathrm{C}\right)$ & Temperature \\
Channel & K-type & Hot bath vein \\
4 & thermocouple & $\left({ }^{\circ} \mathrm{C}\right)$ & - \\
Channel & Infrared sensor & Voltage $(\mathrm{V})$ & \\
5 & & & \\
\hline \hline
\end{tabular}

This test bench is connected to an Agilent data acquisition unit (34972A) with a 20 channels acquisition card (34901A) as multiplexer. Five channels are used for temperature and electrical voltage measurements (cf. Table III) with a sampling frequency of $10 \mathrm{~Hz}$ and a duration of 30 minutes (cf. section Operating protocol).

\section{B. Operating protocol}

To determine this thermal time constant $(\mathrm{k})$, a transient thermal protocol is achieved (cf. Fig. 6).

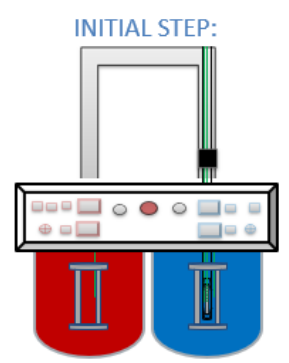

STEP 3:

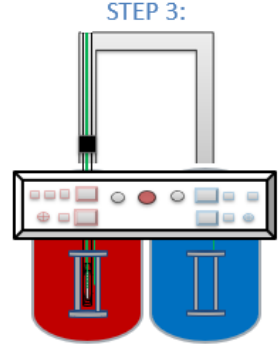

Fig. 6 Diagram of the thermal transient protocol.
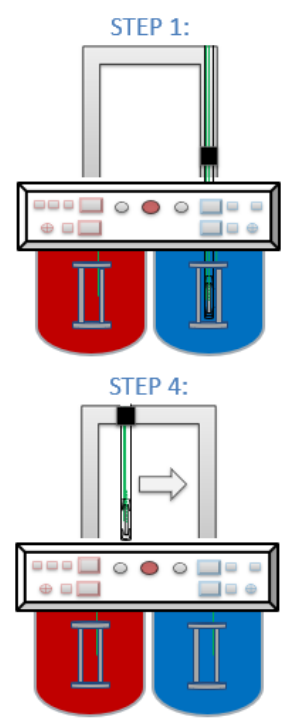
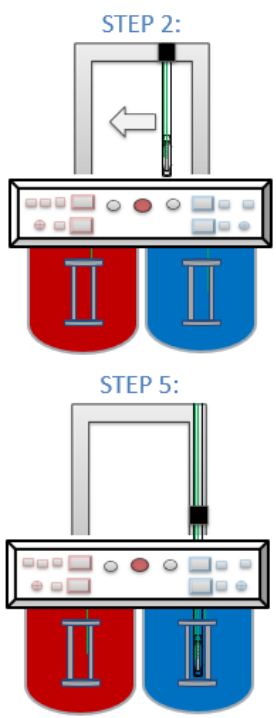

Before starting the test, a specific temperature and velocity of the silicone oil in each thermostatic bath is imposed (initial step), and the calorimeter is immersed in the cold vein. The imposed temperatures in the cold bath and the hot bath can be from $25{ }^{\circ} \mathrm{C}$ to $37{ }^{\circ} \mathrm{C}$ and from $170{ }^{\circ} \mathrm{C}$ to $250{ }^{\circ} \mathrm{C}$ respectively. The rotation speed of the propeller is between $500 \mathrm{rpm}$ to 1250 $\mathrm{rpm}$. After reaching a steady thermal state, data acquisition is started. The calorimeter remains in the cold vein for 10 minutes (step 1) to acquire its initial steady response. Then the mechanical rail quickly moves the calorimeter to the hot vein: heating phase displacement (step 2). The calorimeter remains 10 minutes in the hot vein (step 3) to observe its transient response for a heating phase and to reach a second steady thermal state inside the sensor. The reverse transfer of the calorimeter is then performed, from the hot vein to the cold vein: cooling phase displacement (step 4). Finally, the calorimeter remains for 10 minutes in the cold vein in order to observe its transient 
response for a cooling phase and to reach a final thermal equilibrium (step 5).

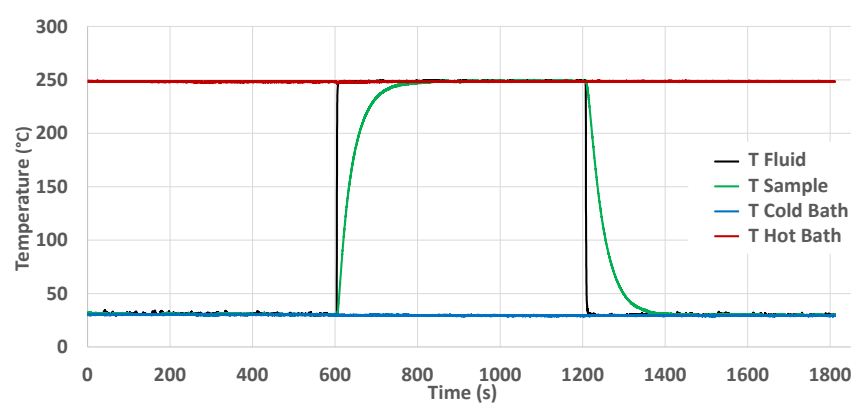

Fig. 7 Temperatures versus time for a test with a propeller velocity of $1000 \mathrm{rpm}$ and with hot and cold temperatures of $250{ }^{\circ} \mathrm{C}$ and of $31^{\circ} \mathrm{C}$ respectively.

Figure 7 gives the temperature measurements versus time for a test carried out with KAROLINA calorimeter by applying this operating protocol with a propeller velocity of $1000 \mathrm{rpm}$, a hot and cold temperatures of $250{ }^{\circ} \mathrm{C}$ and of $31{ }^{\circ} \mathrm{C}$ respectively. The different successive phases (steady and transient) can be observed.

\section{RESULTS}

A test campaign took place from July 9 to July 23, 2020. The purpose of this campaign was to study the evolution of the value of the thermal time constant $(\mathrm{k})$ according to the hot bath temperature, the cold bath temperature, the rotation speed of the propeller inside each bath and the cooling and heating phases. This section focuses first on a brief description of the test campaign. Then the repeatability of the results obtained for the tests is presented. Finally, the influence of each parameter is quantified with an Hadamard matrix.

\section{A. Test campaign}

In order to define the influence of the parameters, it was chosen the extreme temperatures of each range and a median value:

- 3 temperatures for the hot bath: $170,210,250{ }^{\circ} \mathrm{C}$

- 3 temperatures for the cold bath: $25,31,37^{\circ} \mathrm{C}$

Regarding the speed of rotation of the propeller, it is necessary to have a sufficient high speed to be able to homogenize the baths, so the studied speed range is from $500 \mathrm{rpm}$ to $1250 \mathrm{rpm}$ and four values were tested:

- 4 speeds of the rotation of the propeller: 500, 750, 1000, 1250 rpm

By taking these conditions into account, the test campaign was composed of 35 tests. For each test, the values of the thermal time constant $\mathrm{k}$ are determined by applying the method corresponding to the equation (7). This presents a number of 140 values of the thermal time constant $(\mathrm{k})$ (4 values per test: 2 values for the heating phase and 2 values for the cooling phase). Figure 8 gives the distribution of the values. This histogram shows a difference in results of $\mathrm{k}$ between the heating and cooling phases.

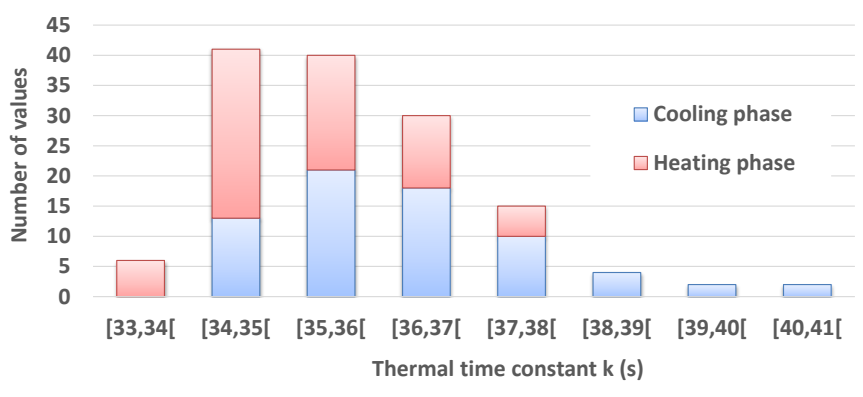

Fig. 8 Distribution of $\mathrm{k}$ values.

The difference between the lower value and the higher value is $7.38 \mathrm{~s}$ with $k_{\min }=33.11 \mathrm{~s}$ and $k_{\max }=40.49 \mathrm{~s}$. The average is $\bar{k}=35.77 \mathrm{~s}$, and the standard deviation is $\sigma_{k}=1.35 \mathrm{~s}$.

\section{B. Repeatability study}

A repeatability study was realized by fixing specific experimental conditions (hot bath at $210{ }^{\circ} \mathrm{C}$, cold bath at $31^{\circ} \mathrm{C}$, propeller rotation speed at $1000 \mathrm{rpm}$ ) and for four successive experiments as described in Table IV.

TABLE IV: REPEATABILITY TESTS

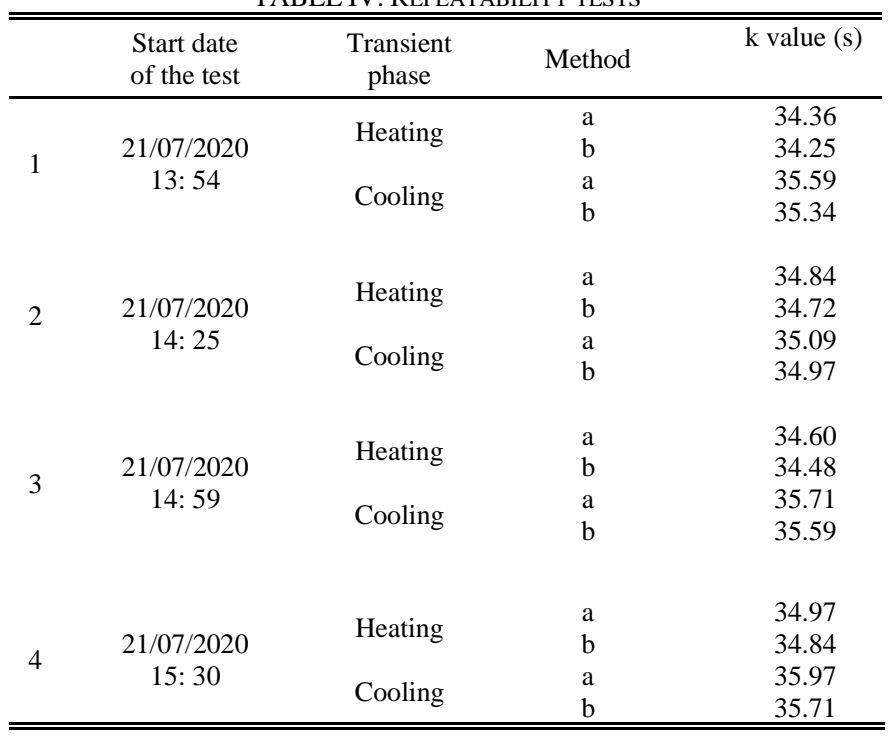

In order to have repeatability conditions, it is necessary to consider separately the results obtained during the heating phase and those obtained during the cooling phase.

A good repeatability is obtained for each phase: $\bar{k}_{\text {heating }}=$ $34.6 \mathrm{~s}, \quad \sigma_{k_{\text {heating }}}=0.25 \mathrm{~s}$ and $\bar{k}_{\text {cooling }}=35.5 \mathrm{~s}$, and $\sigma_{k_{\text {heating }}}=0.34 \mathrm{~s}$. The study confirms the discrepancy between the values of the thermal time constant obtained for a heating phase and those for a cooling phase. This behavior can be explained by a different thermal resistance in the two cases. The method of determination of the k-value (a thanks to the slope or $b$ thanks to the $y$-intercept) does not affect the value of $\mathrm{k}$ significantly (for instance for the heating phase the difference of the two mean values of $\mathrm{k}$ is about $0.1 \mathrm{~s}$ with a similar standard deviation of $0.25 \mathrm{~s}$ ). Consequently, a global study could be carried out by considering the $\mathrm{k}$ value whatever the applied method. 


\section{Influence factors}

It is necessary to identify the factors that have an influence on the value of k. 5 factors were identified with more or less significant influence on the value of the thermal time constant (k). Three of these parameters are linked to the external boundary conditions: hot bath temperature, cold bath temperature, rotation speed of the propellers. Whereas the two last parameters are due to the phase and the data processing respectively: Transient phase (cooling or heating) and the determination method (slope or y-intercept).

The experimental results can be analyzed by using an Hadamard matrix which allows the identification of which factors have the most influence.

For the definition of the matrix, the experiments are considered with factors at 2 levels, the most extreme possible, in order to increase their influence. Table $\mathrm{V}$ gives the factors and their values for the two levels.

\section{TABLE V: INFLUENCE FACTORS}

\begin{tabular}{cccc}
\hline \hline & Factor & Low level & High level \\
\hline $\mathrm{U}_{1}$ & Hot bath temperature & $170{ }^{\circ} \mathrm{C}$ & $250^{\circ} \mathrm{C}$ \\
$\mathrm{U}_{2}$ & Cold bath temperature & $25^{\circ} \mathrm{C}$ & $37^{\circ} \mathrm{C}$ \\
& Propeller rotation & $500 \mathrm{rpm}$ & $1250 \mathrm{rpm}$ \\
$\mathrm{U}_{3}$ & speed & Cooling & Heating \\
$\mathrm{U}_{4}$ & Transient state & Slope & y-intercept \\
$\mathrm{U}_{5}$ & Determination method & & \\
\hline \hline
\end{tabular}

The obtained Hadamard matrix is given in Table VI.

TABLE VI: HADAMARD MATRIX

\begin{tabular}{|c|c|c|c|c|c|c|}
\hline & $\mathrm{U}_{1}$ & $\mathrm{U}_{2}$ & $\mathrm{U}_{3}$ & $\mathrm{U}_{4}$ & $\mathrm{U}_{5}$ & $\mathrm{k}$ values (s) \\
\hline & 1 & -1 & -1 & 1 & -1 & 36.50 \\
\hline & 1 & 1 & -1 & -1 & 1 & 40.16 \\
\hline & 1 & 1 & 1 & -1 & -1 & 35.84 \\
\hline & -1 & 1 & 1 & 1 & -1 & 35.44 \\
\hline & 1 & -1 & 1 & 1 & 1 & 34.12 \\
\hline & -1 & 1 & -1 & 1 & 1 & 35.97 \\
\hline & -1 & -1 & 1 & -1 & 1 & 34.01 \\
\hline & -1 & -1 & -1 & -1 & -1 & 36.90 \\
\hline$b_{i}$ & 6.30 & 3.88 & -12.1 & -6.87 & 1.59 & \\
\hline
\end{tabular}

By applying this matrix coupled with a Pareto analysis defined by equation (8) the influence in $\%$ for each factor is obtained (cf. Fig. 9).

$$
P_{i}(\%)=100 \times \frac{b_{i}{ }^{2}}{\sum b_{i}{ }^{2}}
$$

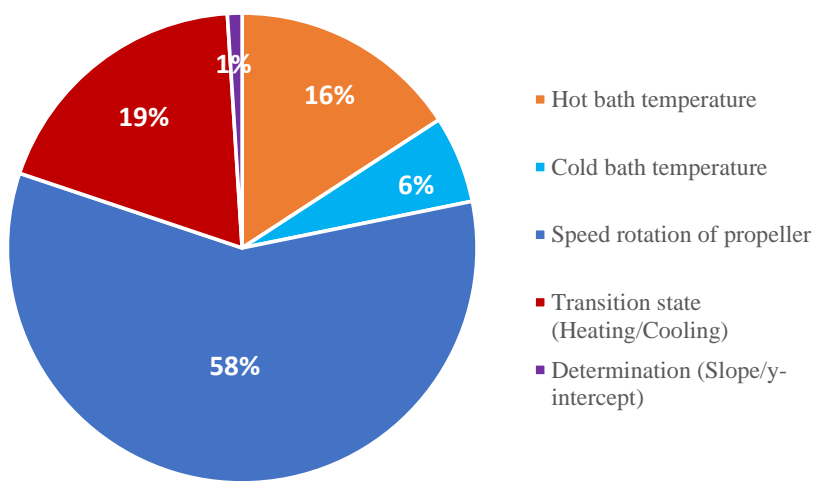

Fig. 9 Quantification of the influence of each factor.

The factor that mainly influences the value of $\mathrm{k}$ is the speed of rotation of the propeller, thus the velocity of the fluid around the sensor in the bath vein. Indeed the increase of the propeller speed leads to the increase of the thermal exchange coefficient (h; $\left(\mathrm{W} \cdot \mathrm{m}^{-2} \cdot \mathrm{K}^{-1}\right)$ ) and consequently the decrease of the thermal resistance between the sample and the fluid. Two other factors also have a significant influence: the transient state (heating/cooling) and the hot bath temperature.

On the opposite, the determination method of $\mathrm{k}$ has no influence, thus this factor can be neglected. For the next campaign, an average between the two values obtained by the two methods could be calculated.

\section{CONCLUSIONS AND OUTLOOKS}

A first experimental study was carried out to determine the thermal time constant of the Polish KAROLINA single-cell calorimeter thanks to a specific thermal transient protocol applied in the BERTRAN bench reproducing reactor conditions (temperature, heat exchange, geometry). This thermal time constant is a key value for the calibration of the sensor and thus for the determination of its sensitivity. The experimental campaign under laboratory conditions confirmed the repeatability of the calibration protocol and determined the influence of five parameters by using an analysis involving Hadamard matrix. The thermal time constant value is independent of the two calculation methods. But it depends on the cooling and heating phase. The temperature of the hot bath also impacts the thermal time constant value. These results and approach will be used to define new test campaign conditions and determine the sensor sensitivity and its uncertainties. Moreover, the instrumentation of the bench will be improved with the adding of a flowmeter in order to determine the fluid velocity $\left(\mathrm{m} . \mathrm{s}^{-1}\right)$ near the calorimeter during the calibration. This quantity is crucial to interpret the results and to compare them with numeric results obtained thanks to a 1-D theoretical model and thanks to 3-D thermal simulations.

The optimized bench will be used for a next test campaign focusing on other calorimeters targeted for a future irradiation campaign within the framework of the CALOR-I project (such as a gamma thermometer or a CALORRE differential calorimeter). 


\section{ACKNOWLEDGMENT}

"The CALOR-I project leading to this publication has received funding from the Excellence Initiative of Aix-Marseille University - A*Midex, a French "Investissements d'Avenir" programme".

\section{REFERENCES}

[1] C. Reynard-Carette, G. Kohse, J. Brun, M. Carette, A. Volte, A. Lyoussi, "Review of nuclear heating measurement by calorimetry in France and USA", Proc. ANIMMA 2017, Jun. 2017, EPJ Web of Conferences 170, 04019 (2018).

[2] J. Brun, C. Reynard-Carette, A. Lyoussi, C. De Vita, M. Carette, M. Muraglia, D. Fourmentel, P. Guimbal, J.-F. Villard, "Comparison of the thermal calibration response of two calorimetric cells dedicated to nuclear heating measurements", IEEE Trans. Nucl. Sci., vol. 61, no. 4, pp. 22542261, Aug. 2014.

[3] A.Volte, C. Reynard-Carette, A. Lyoussi, J. Brun, M. Carette, "Study of the Response of a New Compact Calorimetric Cell for Nuclear Heating Rate Measurements", IEEE Trans. Nucl. Sci., vol. 65, no. 9, pp. 24612470, Sept. 2018.

[4] A.Volte, J. Brun, A. Lyoussi, M. Carette, C. Reynard-Carette, "Qualification of a New Differential Calorimeter Configuration Dedicated to Nuclear Heating Rates up to $20 \mathrm{~W} . \mathrm{g}^{-1}{ }^{1}$, IEEE Trans. Nucl. Sci., vol. 67, no. 11, pp. 2405-2414, Nov. 2020.

[5] R. Van Nieuwenhove, L. Vermeeren, "Nuclear heating measurements by gamma and neutron thermometers", Proc. ANIMMA 2019, Jun. 2019, EPJ Web of Conferences 225, 04003 (2020).

[6] J. Brun, M. Tarchalski, C. Reynard-Carette, K. Pytel, A. Lyoussi, J. Jagielski, D. Fourmentel, J-F. Villard, M. Carette, "Responses of SingleCell and Differential Calorimeters: From Out-of-Pile Calibration to Irradiation Campaigns," IEEE Trans. Nucl. Sci., vol.63, no. 3, pp. 1630 1639, Jun. 2016.

[7] A. Luks, K. Pytel, M.Tarchalski, N. Uzunow, T. Krok, "Modelling of thermal hydraulics in a KAROLINA calorimeter for its calibration methodology validation", NUKLEONIKA, vol 61, no. 3, pp 453-460, Oct. 2016.

[8] J. Brun, C. Reynard-Carette, M. Carette, A. Lyoussi, A. Volte, M. Tarchalski, R. Prokopowicz, "Calibration of a Single-Cell Calorimeter in a New Transient state Test Bench", Proc. ANIMMA 2019, Jun. 2019, EPJ Web of Conferences 225, 04009 (2020).

[9] M. Tarchalski, K. Pytel, P. Siréta, A. Lyoussi, J. Jagielski, C. ReynardCarette, C. Gonnier, G. Bignan, "Principle of calibration of the simple calorimeter for nuclear heating measurements in MARIA reactor and transposition to the case of JHR reactor", Proc. ANIMMA 2013, Jun. 2013.

[10] M. Tarchalski, "Nuclear heating measurements in the Maria reactor and implementation of neutron and photon calculation scheme", Ph.D manuscript, Aix-Marseille Université, Marseille, France, 2016. 